\title{
„Wir initiierten die meisten klinischen Phase-III-Studien“
}

\author{
Protein Powerhouse nennt sich die Forschungs-Schmiede der dänischen Pharma-Firma Novo \\ Nordisk. Seit 1923 ist sie auf Antidiabetika spezialisiert und hat mit ihrem breiten Angebot mo- \\ derner Insuline die führende Position auf dem Weltmarkt. Woran Novo Nordisk aktuell forscht \\ und welche Innovationen demnächst zu erwarten sind, erläuterte Diabetes-Forschungsleiter \\ Prof. Peter Kurtzhals im Gespräch mit SpringerMedizin.
}

\begin{abstract}
Novo Nordisk ist Weltmarktführer im Diabetes-Bereich. Was ist das Erfolgsrezept?

Kurtzhals: Unsere Kernkompetenz ist die Entwicklung von komplexen Proteinen. Wir konzentrieren uns dabei auf wenige Prinzipien. Nach unserer Überzeugung sind die wirksamsten therapeutischen Mechanismen der Ansatz am Insulinund am GLP-1-Rezeptor. Auf diesen Feldern forschen wir. Ein weiterer Fokus sind moderne Applikationssysteme sowie neue Applikationsrouten, um dem Patienten die Therapie zu erleichtern.
\end{abstract}

Wie sichern Sie Ihre Position für die Zukunft? Kurtzhals: Wir investieren jährlich knapp 1 Mrd. Euro, um für Menschen mit Diabetes bessere Therapien zu entwickeln und so die Krankheitsprogression zu verlangsamen. Von 2005 bis 2010 hat Novo Nordisk die größte Zahl an Protein-Patenten in der Diabetologie angemeldet. Wir haben die meisten klinischen PhaseIII-Studien initiiert, aktuell sind es 93. Die Publikationen unserer Forschungsergebnisse wurden im Vergleich zu Mitbewerbern am häufigsten zitiert. Das spricht für die Qualität unserer Forschung.

Welche Innovation dürfen Ärzte und Patienten als nächstes erwarten?

Kurtzhals: Seit etwa fünf Jahren entwickeln wir klinisch das neue Basalinsulin Degludec. Die Phase-III-Studien sind abgeschlossen, die Empfehlung der Europäischen Arzneimittelagentur ist da, sodass die Zulassung Ende 2012 möglich ist.

Welche Vorteile hat Degludec gegenüber den vorhandenen Langzeitinsulinen?

Kurtzhals: Der Hauptvorteil ist, dass das Risiko nächtlicher Hypoglykämien um $25 \%$ oder mehr erniedrigt ist. Als Insulinglargin eingeführt wurde, reduzierte es das Hypoglykämierisiko auch um ca. $25 \%$ gegenüber Humaninsulin. Ein weiterer Vorteil ist, dass sich die Flexibilität des Patienten erhöht: Er kann sein Ba- salinsulin zu jeder Tageszeit spritzen. Wir werden empfehlen, dass Degludec immer zur gleichen Zeit injiziert wird, aber die Flexibilität ist praktisch für $\mathrm{Pa}$ tienten, die viel unterwegs sind, in Schichten arbeiten, unregelmäßige Tagesabläufe haben. Wenn der Patient die Injektion einmal vergisst, ist das nicht so schlimm. Grund ist die mit 24 Stunden viel längere Halbwertszeit versus Glargin oder Detemir.

Sie erforschen darüber hinaus Wege, die Spritze zu vermeiden.

Kurtzhals: Richtig. Ein erheblicher Anteil der Diabetiker zeigt Widerstände gegen Spritzen und eskaliert seine Behandlung deshalb sehr spät. Novo Nordisk forscht daher mit einem großen Team und mit einem signifikanten Budget nach Wegen, Insulin oral verfügbar zu machen. Die Herausforderung besteht darin, lang wirksame Substanzen zu entwickeln, die im Gastrointestinaltrakt stabil bleiben und resorbiert werden. Wir haben Substanzen bereits in klinischer Entwicklung.

Auf eine inhalative Applikation setzen Sie nicht mehr?

Kurtzhals: Nein. Hier gab es ein Produkt auf dem Markt, das für die Patienten sehr umständlich zu bedienen und mit Sicherheitsbedenken behaftet war, Stichwort Lungentumoren. Auch Novo Nordisk erforschte ein inhalatives Insulin, dessen Entwicklung gestoppt wurde. Die Insulin-Tablette ist für den Patienten sehr bequem. Ob sie unbedenklich ist, wenn im Darm lokal hohe Insulinkonzentrationen vorherrschen, müssen wir untersuchen.

Welche Projekte verfolgt Novo Nordisk auf dem Gebiet der Inkretinmimetika?

Kurtzhals: Hier ist zunächst die LEADERStudie zu erwähnen, eine Langzeitstudie über 5 Jahre mit 9000 Diabetes-Risikopatienten, in der die kardiovaskuläre Sicherheit von Liraglutid (Victoza ${ }^{\circledast}$ ) geprüft
Dr. Peter Kurtzhals, Leiter Diabetes-Forschung und Entwicklung bei Novo Nordisk

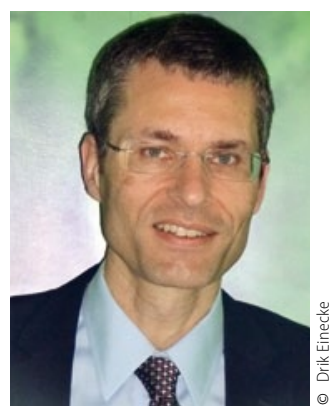

wird. Wir erforschen ein weiteres GLP-1Analogon, Semaglutide, das die Phase-II abgeschlossen hat. Es wird ein Mal wöchentlich injiziert. Auch in klinischer Entwicklung ist ein Projekt mit einem oralen Inkretinmimetikum. Es befindet sich in Phase-I. Und wir testen Liraglutid in etwas höherer Dosis bei Adipositas. Wir haben Daten aus Phase-II, laut denen im Lauf einer 1-jährigen Therapie ein Gewichtsverlust von mehr als 5\% erreichbar ist. Wenn sich die Ergebnisse in der Phase-III bestätigen, können wir die Möglichkeiten einer Markteinführung näher untersuchen. Interview:Dr. med.Dirk Einecke

\section{Novo Nordisk}

Sitz: Bagsværd bei Kopenhagen,

Vermarktung in 190 Ländern,

Mitarbeiter: 33000

Novo Nordisk Deutschland:

Standort in Mainz, gegründet 1958,

ca. 490 Mitarbeiter

Geschäftszahlen:

Umsatz: ca. 9 Milliarden Euro (2011)

F\&E Budget: ca. 1,3 Milliarden Euro (2011)

Gewinn ca. 2,3 Milliarden Euro (2011)

Schwerpunktindikationen:

v.a. Diabetes, daneben: Blutgerinnung, Wachstumshormontherapie, Hormonersatztherapie.

Besonderheit: Die Mehrheit

der stimmberechtigten Aktien

hält die Novo Nordisk-Stiftung

\section{Dr. Peter Kurtzhals}

aktuelle Position: Senior Vice President und Leiter der Diabetes-Forschung und Entwicklung bei Novo Nordisk

Karriere: Professor an der Dänischen Universität für pharmazeutische Wissenschaften, seit 1990 bei Novo Nordisk, Forschungsaufenthalte im Joslin Diabetes Center und an der Harvard Medical School in Boston 